\title{
586 MONITORING THE IMPACT OF MDSC TARGETING DRUGS IN COMBINATION WITH NIVOLUMAB ON ACTIVATION AND PROLIFERATION OF TUMOR RESIDENT EFFECTOR IMMUNE CELLS IN PATIENT- DERIVED 3D-EXPLORE PLATFORM
}

Jared Ehrhart*, Brittany Bunch, Kelly Sussman, Kelly Guzman, Soner Altiok. Nilogen Oncosystems, Tampa, FL, USA

Background Myeloid-derived suppressor cells (MDSCs) represent a diverse population of immature myeloid cells with a strong capacity to suppress the functions of NK cells and CD4+ and CD8+ T-cells in tumor immune microenvironment, thereby reducing the efficacy of immunotherapeutic drugs. MDSC depletion strategies are being investigated to determine if the immunosuppressive effects of MDSCs in the tumor microenvironment can be reduced to improve the efficacy of cancer immunotherapy. Here we employed the 3DExplore ex vivo platform to test the efficacy of MDSC targeting agents alone and in combination with nivolumab using patient derived 3D-tumoroids which retain the tumors' original stroma and suppressive immune landscape.

Methods 3D-tumoroids approximately $150 \mu \mathrm{m}$ in size were generated from fresh patient renal cell carcinoma tumor samples, which were obtained with informed consent and relevant IRB approval. Tumoroids were treated ex vivo with a Phosphodiesterase-5 inhibitor Tadalafil, an HDAC inhibitor entinostat, a potent steroidal liver $\mathrm{X}$ receptor (LXR) ligand DMHCA or a selective CK2 (casein kinase II) inhibitor TBCA alone and in combination with nivolumab. Flow cytometry, high content confocal analysis and cytokine release assays were performed to monitor treatment-mediated changes in tumor immune landscape and tumor cell killing.

Results Multi-parameter flow cytometry (14 color) analysis demonstrated the heterogeneity of tumor immune cell populations including polymorphonuclear (PMN-MDSC) and monocytic (M-MDSC) MDSCs in different patient tumor samples. Furthermore, we performed flow cytometry analysis to assess treatment-induced changes in NK and effector T-cells activation and proliferation profiles that were further correlated with drug impacts on MDSC, Tregs and myeloid cell populations in the ex vivo treatment groups. Additionally, immune cell functions were assessed by multiplex cytokine analysis and treatment-induced tumor cell killing was quantified by high content confocal imaging.

Conclusions Our data demonstrated that the ex vivo 3DExplore is a clinically relevant platform to identify combination of immunotherapy agents capable of overcoming the unique suppressive environment developed by an individual tumor. Furthermore, the 3D-EXplore platform provides unique insight into the intact tumor immune microenvironment to develop therapeutic strategies to overcome immunosuppressive effects of MDSCs to improve the efficacy of immunotherapeutic agents in cancer.

http://dx.doi.org/10.1136/jitc-2021-SITC2021.586 